\title{
Practical issues and some lessons learned from realization of phase sensitive parametric regenerators
}

\author{
Radan Slavík*, Joseph Kakande, and David J. Richardson \\ Optoelectronics Research Centre, University of Southampton, Southampton, SO17 IBJ, UK \\ *r.slavik@soton.ac.uk
}

\begin{abstract}
Practical issues in pump phase synchronization necessary for coherent all-optical processing are discussed, including feed-forward carrier recovery of phase encoded signals. (C)2011 Optical Society of America

OCIS codes: (060.2320) Fiber optics amplifiers and oscillators, (190.4410) Nonlinear optics, parametric processes
\end{abstract}

\section{Introduction}

The phase sensitive amplifier (PSA) provides different gains to the two quadrature components of an input optical signal's electric field. This results in several interesting features compared with the so-called phase insensitive amplifiers, which amplify both electric field quadrature components equally (an example being the erbium doped fibre amplifier). These features includes potentially noise-less amplification [1], generation of squeezed light that has non-classical amplitude and phase noise distributions, as well as all-optical signal processing thanks to the ability to separately manipulate the two quadrature electric field components. Examples of these signal processing applications include the regeneration of phase encoded signals [2] and phase synchronization of ultra-wide bandwidth signals [5].

The most practical realizations of a PSA so far have been based on $\chi^{3}$ parametric processes such as four wave mixing (FWM) where the signal is forced to undergo a parametric interaction with either one or two other optical fields (generally referred to as the pump(s)). One of the greatest technological challenges lies in the need to synchronize the phases of the interacting waves (signal and pumps) at the PSA input. This is not straightforward in applications where the signal is propagated over long distances (accumulating noise) and needs to be phase synchronized inside the PSA that contains the locally-generated pumps (e.g., free running lasers). Unfortunately, most telecom-based applications fall into this category.

Recently, we realized and rigorously tested a 'black-box' optical regenerator for differential phase encoded phase shift keyed signals (DPSK) [2,3,4]. In this paper, we review and concentrate on the lessons we learned in the process rather than the performance of the device itself. This includes a discussion of techniques underpinning 'black-box' operation of the PSA and our recent work on carrier recovery which is of relevance to a far broader range of optical signal processing of coherent signals.

\section{Main challenges}

Generally, in telecoms one deals with digital amplitude modulated (on-off keying, OOK) or phase (BPSK, QPSK) and phase/amplitude (16-QAM, 64-QAM, etc.) modulated signals. A component of the carrier wave is present in the spectrum of OOK signals and it is thus relatively straightforward to phase synchronize such signals to the pump(s). However, with the exception of the low-noise amplification, these signals would do not profit much from PSA technology, as the phase is only of limited importance in their transmission/detection. Phase and phase/amplitude modulated signals are good candidates for PSA-based processing, however, they do not contain a component of the carrier in their spectra and thus phase synchronization to any pumps is thus less straightforward. This latter feature makes these signals difficult-to-process in general and most published works use either differential processing (comparing two consecutive bits) in which the differential phase information is moved to amplitude, or operate in a system in which the carrier is supplied/transmitted separately. Another possibility is to adopt a particular coding scheme that leaves a trace of the carrier [7]. We have recently published another method that operates in a black-box manner (only carrier-less data at its input) that is described later [6].

So far, PSA has been implemented in $\chi^{3}$ media using fully degenerate FWM in an interferometric configuration (non-linear loop mirror, NOLM) [8], or in a non-interferometric configuration using (partially) degenerate FWM [2], Fig. 1. In the first configuration, a single phase locked pump beam at the same frequency as the signal is required. In the latter configuration, the phase matching condition requires $2 \phi_{\text {DataCarrier }}-\phi_{\text {Pump } 1^{-}} \phi_{\text {Pump } 2}=$ const . It is perhaps worth mentioning that fulfilling this condition is not phase-locking in the classical sense in which any pair or signals are phase locked (e.g. $\phi_{\text {DataCarrier- }}-\phi_{\text {Pump } 1}=$ const, $\phi_{\text {DataCarrier }}{ } \phi_{\text {Pump } 2}=$ const $)$.

In our work, we consider only situations in which the incoming signal is contained in a single channel (we do not transmit a pair of signal-idlers carrying the same information over the network, as suggested in [1], Fig. 1b), and 
that the processed signal should have the same carrier frequency (wavelength) as the incoming signal. This can be achieved in the NOLM based approach, or using two-pump degenerate FWM (Fig. 1a, 1c respectively).

a)

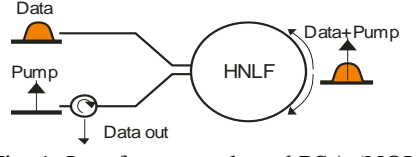

b)

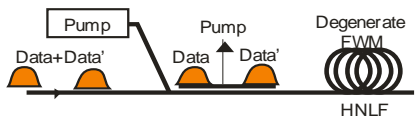

c)

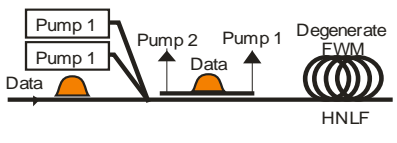

Fig. 1 Interferometer-based PSA (NOLM) (a) and non-resonant based PSA using single-pump non-degenerate FWM (b) and dual-pump degenerate FWM (c) configurations.

\section{Synchronization of incoming data to pumps}

We consider two classes of methods. The first one recovers the carrier wave (a Carrier Recovery, CR) that can be used to further synchronize the pumps. The latter directly synthesizes the pumps thereby fulfilling the phase synchronization condition specified earlier. CR can be achieved using a feed-back [9], or a feed-forward method [10]. Here, we discuss a new feed-forward method we published recently [6] that uses only relatively slow speed electronics. The other class of phase synchronization methods are what we have used in most of our PSA work to date (including all-optical regeneration of binary PSK (BPSK) and QPSK modulated signals): specifically, FWM combined with injection locking of a semiconductor laser.

The first step is common to both methods discussed - modulation stripping as shown in Fig. 2.

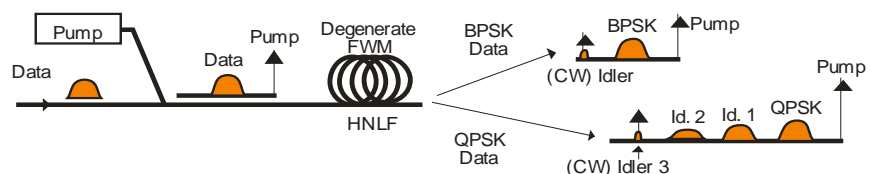

Fig. 2 Modulation stripping - principle shown on an example of BPSK and QPSK modulation formats [12].

Although the idlers of interest (e.g., $1^{\text {st }}$ for BPSK and $3^{\text {rd }}$ for QPSK) are data modulation free, they are not directly useable. Firstly, the phase-stripped idler contains the residual amplitude modulation present on the data; Further reasons are different for the two schemes.

Carrier Recovery: The idlers, Fig. 2 possess phase fluctuations originating from the finite linewidth of the pump laser. Further, the idler wave does not follow the signal carrier itself, but rather its $(\mathrm{M}-1)^{\text {th }}$ multiple. Finally, the idler wave is at a different wavelength, which is undesirable for most applications. (e.g., it cannot be used for homodyne processing or in NOLM). We explain our method on BPSK modulated signals as its extension to higher modulation formats is straightforward.

First, we perform the afore-mentioned modulation stripping together with a second parallel mixing process in which another CW signal (Intradyne LO) is mixed with a portion of the same Pump. The offset frequency between the Intradyne LO and the data carrier is set such that the difference is within the bandwidth of RF electronics.

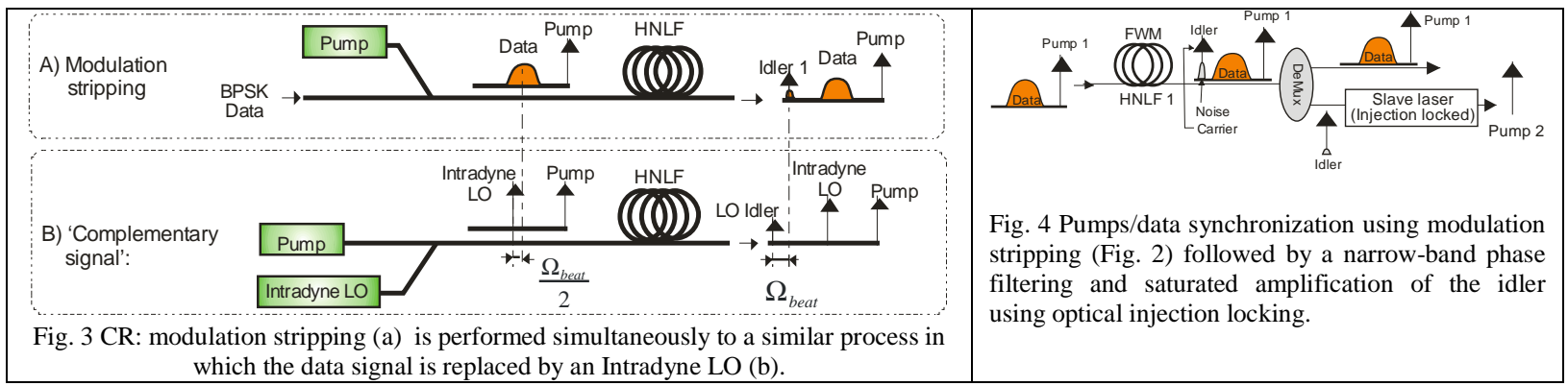

By filtering the two idlers and beating them at the detector, we obtain $\Omega_{\text {beat }}$ (Fig. 3) that can be divided in an RF frequency divider. After some mathematical manipulation it can be shown that [6]: $\omega_{\text {carrier }}=\Omega_{\text {beal }} / 2+\omega_{\text {intradyneLO}}$. Thus, the carrier (Homodyne LO) can be obtained by shifting the Intradyne LO frequency by RF frequency $\Omega_{\text {beal }} / 2$ (using, e.g., a single-sideband modulator). We implemented this method for BPSK signals and recovered carrier at its original frequency with precision better than $1 \mathrm{~Hz}$. The processing bandwidth is practically unlimited as it is based on ultra-broadband FWM. We demonstrated results up to 20 Gbaud, even in the presence of residual dispersion (50 $\mathrm{km}$ of SMF-28). 
Pump-Pump-Data phase synchronization: The scheme shown in Fig. 1 was used for Pump-Data synchronization for many PSA studies [1,2]. The biggest disadvantage is that the idler (Pump 2) has typically much less power than Pump 1 and ensuring power equalization is energetically inefficient and leads to significant noise pick-up during the post-amplification process. An option to mitigate the power issue is to selectively amplify the idler itself via a narrow-band filtering process. When this amplification is also saturated, the amplitude variations due to the residual data amplitude modulation can be also suppressed. Both these conditions can be met by injection locking a semiconductor laser with the idler [11], which is the approach we chose, Fig. 4. The injection locking has another important feature that is crucial for all-optical regeneration: its bandwidth (bandwidth over which it can track phase changes of the idler) can be controlled via the injected power level. If the bandwidth is too large more noise is transferred onto the Pump 2. On the other hand, if the bandwidth is too narrow, phase slips (defined as a slip of phase of $2 \pi$ ) can be observed.

\section{Other practical considerations}

There are two other key issues to be considered for a practical PSA. First, due to the potential for thermal drift/acoustic pick up, it is essential it to ensure that the phase synchronized waves (data, pumps) propagate through as much of a common path as possible and that where the beams are functionally required to travel separately the associated optical path lengths are minimized and are subject to a common environment (e.g. by placing fibers next to each other, or by using photonic integration). Even then though, it is still necessary to implement an active control scheme to control the relative path length e.g., via a PZT fiber stretcher. A feedback speed in the $\mathrm{Hz}$ to several $\mathrm{kHz}$ range (depending on implementation) is usually sufficient. A second key important issue is the choice of the nonlinear medium - particularly with regards to Stimulated Brillouin Scattering (SBS). Pump phase dithering is possible, but requires special configurations [13]. A much preferred method is to use a high-SBS threshold fiber (we have used gradient-strained alumino-silicate nonlinear fibre from OFS, Demark [14]), or another non-linear medium, including $\chi_{2}$ media such as PPLN [15], offering far higher SBS thresholds.

\section{Conclusions}

Ensuring proper phase synchonization between all participating beams (signal and pumps) is a pre-requisite in any coherent all-optical signal processing system. We have reviewed some of our recent work on this issue as well as some other practical issues related to FWM as an example of such optical coherent signal processing phenomena.

We would like to thank to OFS Denmark for the HNLF; Eblana Photonics, Ireland for the discrete mode lasers; and Adonis Bogris for theoretical support. This research has received funding from the European Communities Seventh Framework Program FP/2007-2013 under grant agreement 255368 (TOP CLASS).

\section{References}

1. Z. Tong et al, "Towards ultrasensitive optical links enabled by low-noise phase-sensitive amplifiers," Nat. Photon. 5, pp. 430-436 (2011).

2. R. Slavík et al, “All-optical phase and amplitude regenerator for next-generation telecommunications systems,” Nat. Photon. 4 , 690-695 (2010)

3. R. Slavík et al, "Coherent All-Optical Phase and Amplitude Regenerator of Binary Phase-Encoded Signals, IEEE Journal of Selected Topics in Quantum Electronics, available on-line, 2011.

4. R. Slavík et al, Field-trial of an All-Optical PSK Regenerator/Multicaster in a 40 Gbit/s, 38 Channel DWDM Transmission Experiment," to appear in J. of Lightwave Technol., Dec. 2011.

5. R. Slavík et al, "Fourier synthesis of transform limited low phase noise, ultrahigh repetition rate short pulses," submitted to OFC 2012.

6. R. Slavík, J. Kakande, and D.J. Richardson, "Feed-forward True Carrier Extraction of High Baud Rate PSK Signals Using Slow Electronics," ECOC 2011, Geneva, paper We.10.P1.59, Sept. 2011.

7. A. Chiuchiarelli, M. J. Fice, E. Ciaramella, and A. J. Seeds, "Effective homodyne optical phase locking to PSK signal by means of 8b10b line coding," Opt. Express 19, 1707-1712 (2011).

8. K. Croussore, et al., "Demonstration of phase-regeneration of DPSK signals based on phase-sensitive amplification," Opt. Express, 13 , 3945-3950 (2005)

9. K. Kim et al, “All-Optical Carrier Synchronization Using a Phase-Sensitive Oscillator,” Photon. Tech. Lett. 19, $987-999$ (2007).

10. S.K. Ibrahim et al., "Novel Carrier Extraction Scheme for Phase Modulated Signals Using Feed-Forward Based Modulation Stripping," European Conference on Optical Communications (ECOC), 19-23 Sept. 2010, Torino, Italy, paper We7A4.

11. R. Phelan, B. Kelly, J. O'Carroll, C. Herbert, A. Duke and J. O' Gorman, “- $40^{\circ} \mathrm{C}<\mathrm{T}<95^{\circ} \mathrm{C}$ mode-hop free operation of an uncooled AlGaInAs-MQW discrete-mode laser diode with emission at $\lambda=1.3 \mu \mathrm{m}$.” Electron. Lett. 45, 43-45 (2009).

12. J. Kakande et al, "First demonstration of all-optical QPSK signal regeneration in a novel multi-format phase sensitive amplifier," ECOC 2010 Turin 19-23, Italy, PD 3.3, Sep 2010.

13. J.Kakande, R.Slavík, F.Parmigiani, P.Petropoulos, D.J.Richardson, "Synthesis of phase-locked counter-phase modulated pumps for SBSsuppressed fiber parametric amplifers,” CLEO/QELS 2010, San Jose, 16-21 May 2010.

14. L. Grüner-Nielsen et al., "A Silica Based Highly Nonlinear Fibre with Improved Threshold for Stimulated Brillouin Scattering," Tu.4.D.3, ECOC 2010 Turin 19-23, Italy, Sep 2010.

15. B.J. Puttnam, D. Mázroa, S. Shinada, and N. Wada, Experimental Investigation of Phase Squeezing in a Non-Degenerate PSA Based on a PPLN Waveguide," ECOC 2011, Geneva, paper Tu.5.LeSaleve.2, Sept. 2011. 